\title{
Actitudes Éticas de los estudiantes y egresados en carrera de medicina con metodologías activas
}

\author{
Ethical Attitudes of Brazilian Medical Students and \\ Graduates with Active Methodologies
}

\author{
Maria Rita Carvalho Garbi Novaes ${ }^{\mathrm{I}}$ \\ Luiz Carlos Garcez Novaes ${ }^{\mathrm{I}}$ \\ Dirce Guilhem ${ }^{\text {II }}$ \\ Fernando Lolas Stepke ${ }^{\text {III }}$ \\ Carla Cristina Costa Silveira ${ }^{\mathrm{I}}$ \\ Ricardo Shoiti Komatsu ${ }^{\mathrm{IV}}$ \\ Eliane Mendonça Vilar Trindade ${ }^{\mathrm{I}}$ \\ Murilo Galvão Guiotti ${ }^{\mathrm{I}}$
}

PALAVRAS-CHAVE

- Ética

- Bioética

- Carrera de Medicina

- Educación Médica.

\section{KEY WORDS}

- Ethics

- Bioethics

- Medical Studies

- Medical Education

Recebido em: 02/11//2008

Reencaminhado em: 03/05/2009

Aprovado em: 16/06/2009

REVISTA BRASILEIRA DE EDUCAÇÃO MÉDICACA

$4 3 \longdiv { 3 4 ( 1 ) : 4 3 - 5 6 \cdot 2 0 1 0 }$

\section{A B STR A C T}

The objective of this study was to conduct a diagnosis of the comprehensive inclusion of ethics in Brazilian medical training with a problem-based learning methodology and to describe students' and graduates' perceptions of ethical attitudes. The methodological design was a descriptive and documental case study with a qualitative and quantitative approach. The sample consisted of 20 students per course year, totaling 120 students and 40 alumni from two graduating classes at the ESCS School of Medicine. The project was approved by the Institutional Review Board of the State Health Secretariat, Federal District, Brazil. ESCS students and graduates showed that they approach ethical conflicts and respect for patients. However, an analysis of ethical sensitivity revealed weak perceptions and inappropriate attitudes by medical students, especially in the early years of medical school, requiring more systematic discussions on ethical and bioethical aspects integrated with practical activities, in order to increase and strengthen ethical reflection by students.

\footnotetext{
${ }^{I}$ Escola Superior de Ciências da Saúde, Brasília, DF, Brasil..

II Universidade de Brasília, Brasília, DF, Brasil.

IIUniversidad de Chile, Santiago, Chile; Organización Panamericana de Salud. Programa Regional de Bioética, Santiago, Chile.

IV Faculdade de Medicina de Marília, Marília, SP, Brasil.
} 


\section{INTRODUCCIÓN}

Actualmente existen 175 cursos de medicina en Brasil, país considerado el $2^{\circ}$ en el mundo en número de escuelas de medicina, superado solamente por India, con 202 cursos en esta area ${ }^{1-4}$.

La ética se insertó en el currículo médico de cursos brasileños a fines del siglo XIX, en asignatura asociada a la medicina legal. Ya la bioética está inserta en la educación médica desde hace ya unos 30 años $^{4,5}$.

Diversos eventos e instituciones han considerado la necesidad de un cambio efectivo en el proceso educativo de los estudiantes de medicina. Los modelos de enseñanza innovadores se contraponen a los modelos tradicionales. Del estudio de estos modelos se identifican las teorías de Flexner $(1910)^{6}$, que presenta teorías con los objetivos curriculares establecidos previamente, selección y direccionamiento de las experiencias, utilizadas actualmente por muchas escuelas médicas. Para Tyler ${ }^{7}$ el currículo es "básicamente lo que ocurre a los alumnos en la escuela como consecuencia de la actuación de los profesores". Taba ${ }^{8} \mathrm{co}-$ mienza a introducir el diagnóstico de necesidades sociales como parte esencial del diseño. Establece explícitamente el vínculo escuela - sociedad, definiéndose el currículo como "[...] un documento que planifica el aprendizaje".

En 1988 se llevó a cabo la Conferencia Mundial de Educación Médica en la ciudad de Edimburgo, Escocia. A través de la "Declaración de Edimburgo", se ha recomendado que el desarrollo de competencias profesionales debería estar asociado al reconocimiento de valores sociales culturales y éticos, además de promover la interacción entre las escuelas de medicina y los servicios básicos y comunitarios de salud ${ }^{4,5,8,9}$.

En 1991 se formó la Comisión Interinstitucional de Evaluación de la Enseñanza Médica - CINAEM, que asumió un rol de carácter nacional en la articulación de técnicas y políticas con el objetivo de iniciar un movimiento para la reforma de la enseñanza del médico. Este movimiento posibilitó que 76 de las 80 Escuelas Médicas existentes en esa época en el país evaluasen no sólo el modelo pedagógico utilizado, sino también los recursos humanos y el perfil del alumno egresado que ellos buscaban ${ }^{9-12}$.

En Brasil, se ha destacado el incentivo de la Asociación Brasileña de Educación Médica - ABEM, en la reformulación de la educación médica. Otras iniciativas también fueron desarrolladas por la Organización Mundial de la Salud - OMS ${ }^{3}$. Las principales directrices internacionales, entre las cuales Learning Objectives for Medical Student Education Association of American Medical Colleges, de 1998, de los Estados Unidos de América ${ }^{12}$ y Tomorrow`s doctors of General Medical Council ${ }^{13}$, además de las diversas Guías de la Association for Medical Education in Europe ${ }^{14}$, todas re- comiendan objetivos claros y legitiman las bases de una orientación curricular dirigida al aprendizaje de conocimientos y el desarrollo de habilidades éticas y humanistas en un contexto de profesionalismo.

En Brasil, las Directrices Curriculares para el pregrado en Medicina, aprobadas por el Colegio Brasileño de Medicina y por el Ministerio de Educación en 2001, legitimaron las bases para la actual formación médica orientada a la adquisición de conocimientos generales, éticos, humanistas, con la perspectiva de la integralidad de la asistencia, responsabilidad social y compromiso con la ciudadanía ${ }^{3}$.

En 2002, el Ministerio de Salud y el Ministerio de Educación instituyeron el Programa Nacional de Incentivos a Cambios Curriculares en los Cursos de Medicina - PROMED. Este programa tiene por objeto incentivar las escuelas de medicina brasileñas a insertar cambios pedagógicos significativos en los currículos de los cursos de medicina, empleando metodologías activas de enseñanza ${ }^{15}$.

La interacción de los diversos dilemas de la práctica de la medicina moderna ha llevado a la reformulación de la función de la ética en la carrera médica. La expansión del conocimiento médico, evidenciado por la tendencia a la especialización, ha mejorado la calidad y expectativa de vida, con un costo asociado al deterioro de la relación médico-paciente.

El impacto de la ciencia y la tecnología en la medicina ha promovido nuevos dilemas con soluciones que exigen decisiones también nuevas. La educación médica debe acompañar estos avances.

La formación ética de los estudiantes de medicina representa un tema importante en la educación médica. La enseñanza de la bioética a los estudiantes no puede cimentarse exclusivamente en el paradigma educativo de las competencias. El alumno logrará las competencias para actuar sobre los conflictos éticos de la vida profesional y tomar decisiones que sean éticamente consistentes $^{16-19}$.

Para promover la educación moral, en general y la bioética, en particular, la discusión de dilemas morales puede ser un buen recurso metodológico en una época en que urge desarrollar la capacidad de dialogo entre las personas para resolver problemas sin hacer uso de procedimiento educativo, válido para suscitar el diálogo y adoptar posturas razonadas y razonables en el terreno de los valores, actitudes y conducta ${ }^{19}$.

Las innovaciones metodológicas y curriculares sugieren la imperativa inserción de la ética y un cambio en el proceso de aprendizaje, considerando el compromiso de la escuela con la asistencia a la salud, la valorización del desarrollo de habilidades y actitudes. 
Debe promoverse en el estudiante la lectura del imaginario social que circunscribe las enfermedades, con el objetivo de formar un profesional inmerso en el contexto de la enfermedad ${ }^{5}$.

Frente a esta realidad, se propone un cambio en el estatuto epistemológico de la medicina y la educación médica. Tanto la práctica como la enseñanza de la medicina deben ser integrales en su sentido más amplio, con una concepción del hombre desde una perspectiva que lo encuadre plenamente en la sociedad en la que le toca vivir. Asimismo, se implementan nuevas estrategias pedagógicas destinadas a enfocar los problemas de una manera global, evitando caer en la fragmentación de subespecialidades a la que nos hemos visto expuestos en las últimas décadas ${ }^{20}$.

El perfil de enseñanza de la bioética que se propone debe responder al principio de que respetar no es sinónimo de compartir y de que el respeto por el otro es condición sine qua non de la vida democrática $^{19,22}$.

\section{Estudios antecedentes sobre actitudes éticas en estudiantes de las carreras de salud}

En la Facultad de Medicina de la Universidad de Chile se desarrolló una investigación para medir los cambios de actitudes éticas a lo largo de los estudios de medicina. Una determinante importante fue que la investigación se realizó un año antes de que se implementara en el primer y sexto año el curso de Ética Médica, de modo que la actitud ético-médica de las tres cohortes (alumnos de primer y sexto año, y postgraduados) no se vio influenciada por una exposición formal a materias de bioética ${ }^{23-26}$.

Un estudio en dos universidades de Chile para determinar el nivel de conocimientos de Bioética, a partir de la respuesta a un cuestionario, concluyó que los alumnos no poseen un conocimiento suficiente o por lo menos aceptable sobre bioética ${ }^{25}$.

En el Municipio de Trinidad se realizó un estudio con el personal de enfermería y de un total de 480 mujeres se seleccionaron, aleatoriamente, a 150 para constituir la muestra y aplicarles una encuesta para explorar sus conocimientos sobre los principios de bioética y otros aspectos relacionados con la aplicación de dichos principios. Las primeras cinco preguntas indagaban sobre los principios elementales de bioética y las cinco restantes sobre la ética médica. Los resultados reafirmaron la necesidad de impartir cursos sistemáticos de bioética al personal de enfermería para garantizar una mayor y más humana atención a los pacientes y sus familiares ${ }^{25}$.

En la Escuela de Medicina de la Universidad de Dundee; Rennie y Rudland estudiaron la diferencia de actitudes presentes en los estudiantes de ciencias médicas hacia conductas académicas no correctas, observando diferencias entre los años y los distintos escenarios que reflejan un cambio en las actitudes de los estudiantes conforme avanza su formación ${ }^{28}$.

En la Escuela de Medicina Dental de la Universidad de Tokio, Mayeda y Takaze llevaron a cabo un estudio para medir los conocimientos de ética luego del inicio de las prácticas clínicas de los estudiantes ${ }^{29}$.

En la Universidad de Buenos Aires, Ormart efectuó un estudio acerca de las interacciones sobre nociones de deontología profesional de un grupo de pares, en el contexto del aprendizaje formal de la educación superior ${ }^{30}$.

En la Escuela de Odontología de la Universidad de North Carolina, Mofidi, Strauss, Pitner y Sandler, en 2003, investigaron en una muestra de 160 alumnos los incidentes críticos de dilemas éticos que enfrenta el estudiante de odontología en sus actividades de práctica pre profesional en las comunidades ${ }^{31}$. En un cuestionario aplicado a los estudiantes de Medicina de la Universidad de Dundee se estudió las actitudes hacia la mala conducta académica buscando concluir acerca de la honestidad de los futuros profesionales ${ }^{32}$.

Se estudiaron las percepciones frente a ciertos dilemas éticos de los estudiantes de Medicina cursando entre el segundo y tercer año, a seguir los investigadores elaboraron sus informes sobre los resultados, después de impartir un curso de bioética, encontrando altos puntajes después de la intervención ${ }^{32}$.

En una reunión del Programa Regional de Bioética de la Organización Panamericana de Salud se llegó a la conclusión de que es insuficiente la educación sobre los derechos y deberes éticos en la comunidad científica y la comunidad general, así como no está claro cuáles son los beneficios obtenidos por la comunidad y quién debe "negociar" por ellos. Por lo tanto, una propuesta de acción es difundir, educar y abogar permanente sobre el tema, dirigida a la comunidad general y, sobre todo, a los encargados de la gestión en salud ${ }^{33}$.

\section{Planteamiento del problema}

En situaciones reales, el enfrentamiento de problemas que comprenden aspectos morales y de conflicto se presentan de forma insuficiente, como simplemente un código de ética profesional, que establece lo que es o no permitido hacer. Con frecuencia se observa lo amplias que resultan ser las normas, aunque esas normas sean perfectamente conocidas, así como las leyes y las penalidades resultantes de la infracción ${ }^{1-5,34}$.

Sin embargo, la bioética corrobora la necesidad de trascender los lineamientos deontológico y legal, apoyados en la moralidad, para fortalecer la reflexión ética vinculada a la dimensión biopsicosocial del individuo ${ }^{3-5}$. 
Este problema lleva a creer que la inserción de la ética, bioética y humanización en asignaturas aisladas en el currículo médico, notoriamente pautada en el abordaje simplista del Código de Ética Médica y discutida de forma descontextualizada no estaría de acuerdo con las directrices curriculares actuales.

Actualmente la formación en bioética hace parte de la competencia necesaria del profesional de salud y un objetivo importante es proporcionar elementos de juicio a los futuros profesionales para comprender los debates en bioética que se producen en la sociedad y la realidad social de su país.

Para promover la educación moral, en general, y la bioética, en particular, la discusión de dilemas morales puede ser un buen recurso metodológico ${ }^{17,35}$.

El médico que la sociedad necesita es un médico con sólida formación general, entrenamiento en los diferentes niveles de atención a la salud, formación ética y humanista.

En métodos tradicionales de aprendizaje, la educación médica ha sido desarrollada mediante clases magistrales en las cuales se reúnen en un mismo ambiente muchos alumnos y profesores calificados, con transmisión pasiva de los conocimientos del maestro. ${ }^{16}$ A partir de la observación de este método de enseñanza y por reconocer que este método no atendía plenamente a la formación del profesional que la sociedad necesitaba, se han elaborado propuestas de modificación en las Directrices Curriculares Brasileñas para la carrera de medicina, priorizándose las metodologías activas de enseñanza-aprendizaje, con integración entre los ciclos básico y clínico (integración vertical) y actividades relacionadas con la práctica profesional (integración horizontal) $)^{36-44}$.

Este modelo innovador ofrece la posibilidad del aprendizaje activo, ya que el estudiante aprende en la medida que transforma el objeto del conocimiento con un enfoque interdisciplinario, aplicando métodos propios de la investigación científica y de trabajo en pequeños grupos, e incorpora concepciones de la enseñanza activa crítica y reflexiva, haciendo énfasis en las relaciones de la enseñanza y la sociedad ${ }^{44-48}$.

En la enseñanza activa se integra el contenido de diferentes asignaturas alrededor de un objeto de estudio que se define a partir de un problema concreto y socialmente relevante para la profesión médica. El currículo se estructura sobre la base de los problemas actuales y perspectivos del profesional, integrándose a este la dimensión académica y social ${ }^{48-50}$.

El Curso de Medicina de la ESCS utiliza metodologías activas de enseñanza y aprendizaje tales como la metodología de la problematización, a través del Arco de Maguerez, aprendizaje basado en problemas y conducido en pequeños grupos de tutorías, las actividades prácticas de interacción de la enseñanza con servicios y la comunidad (IESC), de habilidades y actitudes (HA) en semiología y comunicación, desarrolladas a partir del primer año del curso de medicina, con el fin de adquirir competencias ${ }^{39}$.

En 2007 llega a su conclusión el primero ciclo del curso de medicina de la ESCS, con las primeras promociones de médicos (ciclo completo del curso). Este fue el momento que trajo la necesidad de evaluar y discutir el plan pedagógico del curso, para calificar las prácticas educativas, considerándose las necesidades sociales del país y en especial de la capital, Brasilia, Distrito Federal.

A partir de las tendencias y experiencias brasileñas e internacionales en educación médica, surgieron algunas interrogantes que motivaron esta investigación: ¿Es posible enseñar comportamientos y actitudes a los estudiantes de la carrera de medicina? ¿Se puede enseñar comportamientos éticos que respeten la vida y la diversidad del otro? ¿Cómo se lleva a la práctica esta enseñanza? ¿Es adecuado y suficiente el método usado para la inserción de la ética en el Curso de Medicina de la ESCS?

\section{OBJETIVOS}

Desarrollar un diagnostico de la inserción integrada de la ética en la carrera de medicina brasileña con una metodología de aprendizaje basada en problemas y describir las percepciones de actitudes éticas de los estudiantes y egresados de la carrera de medicina de la Escuela de Ciencias de la Salud - ESCS, con el objetivo de promover discusiones de la gestión curricular.

\section{METODOLOGÍA}

\section{Tipo de estudio}

El diseño metodológico es un estudio de caso, descriptivo, con abordaje cualitativo y cuantitativo.

\section{Identificación del escenario y fenómeno sociocultural a estudiar}

\section{Escenario: Población y muestreo}

El universo está constituido por 80 estudiantes/año, haciendo un total de 480 alumnos de la carrera de medicina en seis años.

La muestra de esta investigación está constituida por 20 (veinte) estudiantes / año de la carrera, con un total de 120 estudiantes, de ambos sexos (1:1). Igual proporción es acotada para seleccionar a los egresados del curso. La muestra comprendió a 40 egresados de dos promociones del Curso de Medicina de la ESCS, de ambos sexos (1:1).

\section{Diseño metodológico}

Primera fase de la investigación

En la primera fase de la investigación, se busca analizar la percepción de actitudes éticas de los estudiantes $(n=120)$ y egre- 
sados de dos promociones $(n=40)$ del Curso de Medicina de ESCS, según el año de estudio.

Las entrevistas a los estudiantes se llevaron a cabo en las instalaciones de la facultad, con una previa comunicación y solicitud de permiso a las autoridades y consentimiento informado por escrito de los participantes. Se estableció un cronograma para la recolección de los datos según el nivel de estudios.

Las áreas de interés contempladas en la guía de entrevista fueron las siguientes:

(a) deontología médica y confidencialidad;

(b) ética médica y relación médico-paciente;

(c) procedimientos éticos en la enseñanza e investigación médica (con cadáver);

(d) ética y respeto en la relación multidisciplinaria; considerándose la perspectiva de algunos valores como: información, secreto, respeto, confianza, justicia, autonomía, dignidad, veracidad, consentimiento, compromiso y los principios básicos de la bioética: autonomía, justicia, beneficencia, no maleficencia, sin jerarquización prioritaria de valores. Estos valores se seleccionaron teniendo como base el Código de Ética del Colegio Brasileño de Medicina.

Posteriormente a la aplicación del cuestionario a los estudiantes y egresados, siguiendo la teoría de las representaciones sociales $^{40,41}$, se realizó el análisis de contenido de las expresiones verbales de los estudiantes y egresados, capturando toda la variabilidad posible en los discursos producidos referentes a temas específicos, concernientes a los dilemas éticos de los problemas propuestos, seguido de categorización y descripción de los núcleos de sentido.

Para la discusión escrita e individual de dilemas éticos, descritos a continuación, se utilizó como instrumento una entrevista semidirigida, con respuestas abiertas. De forma a mantener el anonimato de las personas entrevistadas, se cambió el nombre de las mismas con el fin de preparar este informe de resultados. La entrevista a los estudiantes y egresados se llevó a cabo entre los meses de julio y diciembre de 2007.

(a) Durante la consulta, el doctor Juan atiende en la enfermería de molestias infecciosas a un paciente con VIH/SIDA, asintomático. El Dr. Juan, utilizando un lenguaje cercano, le pregunta si le ha revelado a su esposa el diagnóstico. El paciente relata que si su esposa viene y se entera de la verdad acerca de su enfermedad, su matrimonio se acaba. Por lo cual el paciente, pide el acto de secreto al médico. El Dr. Juan le revela el diagnóstico a la esposa del paciente. Comente la actitud del médico.

(b) José, estudiante de internado, cuenta que durante la visita en una enfermería oncológica a un paciente pediátrico, fue- ra de posibilidades terapéuticas, fue reprendido en público por un profesor asistente por haber mantenido un contacto más afectuoso con la madre del niño, con la intención de confórtala ante el pronóstico recibido. Comente la actitud del estudiante y del profesor.

(c) Juan es un interno del último año del curso de medicina y nunca tuvo la oportunidad de desarrollar procedimientos como intubación orotraqueal o punción venosa central. Juan está trabajando en la emergencia del hospital en que cursa su internado. En cierto momento, ingresa un paciente politraumatizado grave que lamentablemente fallece. Ante su inseguridad de adquirir nuevas competencias, Juan aprovecha la oportunidad para practicar esos procedimientos en el cadáver. Durante el período de su práctica, entra en la sala la hija del difunto y le pregunta qué esta haciendo con su padre. ¿Cuál sería la actitud del interno al momento de enfrentar esta situación?

(d) El doctor Jorge obtuvo su título de médico y optó por trabajar como médico en la comunidad de una ciudad del interior. Durante el trabajo, el Dr. Jorge observó que la enfermera ejercía la medicina con consultas de puericultura, cambiaba recetas de medicamentos de diabéticos e hipertensos, además de desarrollar algunos procedimientos como la toma de la prueba cérvicovaginal (Papanicolaou). Durante una reunión interdisciplinaria en la unidad de salud, el doctor Jorge reprende sus actitudes acusándola de incompetente debido a los procedimientos que son exclusivos a la actividad de un médico. Sin embargo, no es atendido. Comente la actitud del Dr. Jorge y cuál sería la mejor manera de manejar la situación.

En la elaboración de la versión definitiva de la entrevista con los dilemas éticos, se contó con la opinión de cuatro expertos, profesores médicos del curso de medicina de la ESCS, los que conformaron un panel de discusión con relación a los temas de interés.

\section{Segunda fase de la investigación}

En la segunda fase de la investigación se realizó la evaluación cuantitativa y descriptiva de la inserción de la ética en el core curriculum de 2001 (año de la implantación del curso) y de 2006, disponibles en versión electrónica.

En el presente estudio se adaptó el concepto del curriculum de las Pautas Mínimas del International Institute of Medical Education, que establece un plan curricular con los objetivos que deberán obtenerse y los métodos para realizar la enseñanza y la evaluación curricular ${ }^{42}$.

Se investigaron 32 descriptores relacionados con la ética obtenidos de los descriptores en Ciencias de la Salud (DeCS/MeSH) en 
los objetivos educacionales y contenido cognitivo de los módulos, habilidades y actitudes e interacción con la comunidad, del 1er a $4^{\underline{ }}$ año y el programa de internado ( $5^{\circ}$ y $6^{\circ}$ años) del Curso de Medicina de la ESCS/FEPECS.

Los términos obtenidos en DeCS/MeSH son: ética, bioética, privacidad, información, secreto, respeto, confidencialidad, confianza, confiabilidad, beneficencia, maleficencia, moral, justicia, autonomía, dignidad, veracidad, fidelidad, distanasia, ortotanasia, eutanasia, consentimiento, deontología, legal (medicina legal), dilemas, conflictos, racionalidad, juicio, norma, ley, conducta, responsabilidad, compromiso.

La evaluación de la integración curricular de este estudio se basó en la metodología SPICES de Harden ${ }^{43}$, modificada por Berg ${ }^{44}$. El primer autor desarrolló una estrategia para la construcción curricular y el segundo para la evaluación y comparación de los currículos. SPICES es un acróstico mnemónico de la lengua inglesa, empleado para designar las características curriculares deseables de acuerdo con las directrices curriculares internacionales: S - Student-centred, P - Problem-based, I - Integrated, C - Community-based, E - Electives, S -Systematic education.

\section{Análisis de los datos}

Los datos cuantitativos se procesaron en el Software estadístico SPSS, versión 15.1., mediante estadísticas descriptiva y analítica. Los resultados se presentaron en gráficos. Las significancias entre las diferencias se desarrollaron mediante el test de hipótesis. El intervalo de confianza acotado fue á=0,05. La probabilidad de significancia se expresó en $p$-value.

Los datos fueron significativos para $\mathrm{p} \leq 0,05$. Intervalo de confianza de 95\% (Test de Correlación de Person).

Para el análisis de triangulación, se cruzaron los datos cualitativos y cuantitativos de las dos fases para detectar una tendencia lógica en la mezcla de los resultados ya que la validez de la triangulación descansa en la capacidad de organizar los materiales en un marco coherente. Una de las prioridades de la triangulación, como estrategia de investigación, es aumentar la validez de los resultados y mitigar los problemas de sesgo ${ }^{39-41}$.

Desde esta perspectiva puede considerarse que una primera manifestación de la misma son las escalas de medida como referencias más validas y fiables. La triangulación de datos supone el empleo de distintas estrategias de recolección de datos. Su objetivo es comparar las tendencias detectadas en un determinado grupo de observaciones con los de otra para analizar si los patrones de comportamiento son similares.

Generalmente se recurre a la mezcla de tipos de datos para validar los resultados de un estudio piloto inicial. La triangula- ción entre métodos ofrece la oportunidad de mejorar el diagnóstico organizativo sintetizando los resultados derivados de la utilización de múltiples métodos científicos en una interpretación válida y coherente ${ }^{40}$.

\section{Consideraciones Éticas}

Este proyecto fue aprobado por el Comité de Ética en Investigación-SES/DF, Brasilia, Brasil (Protocolo: 052/07). Se solicito la participación de los estudiantes y egresados mediante la aplicación del Consentimiento Informado. Se aseguró el sigilo de la información de los estudiantes, egresados e instituciones participantes.

\section{RESULTADOS}

El análisis cualitativo, en la presente investigación, utilizó el análisis de tipo hermenéutico. En este tipo de análisis, la búsqueda de sentido está dada en hacer converger el mundo de quien interpreta y el del autor del discurso, en otras palabras permite lograr un conocimiento acabado del contexto en el cual se produce el discurso sometido a análisis, ya que es imposible abstraerse del contexto de producción del discurso, es decir del entorno social, cultural, político, ideológico, geográfico, en el cual aquellos se producen ${ }^{40}$.

Las actitudes son una 'construcción psicológica' que se explica como una inferencia de la teoría de la conducta y no es un hecho tangible, es un término especulativo que se formula con referencia explícita a fenómenos observables. Las actitudes nos permiten explicar las conductas frente a eventos específicos.

Se analizó y procesó todo lo que fue dicho por los estudiantes y egresados, considerándose que las expresiones verbales en cada artículo mostraron la visión del orden social y la subjetividad, seguida por una captación sistematizada de los aspectos en el tratamiento de fenómenos y representaciones sociales, categorización y elección de los núcleos de sentido con relación a los dilemas éticos propuestos a los estudiantes de diferentes años del curso y egresados, según la descripción de la categorización y los núcleos de sentido presentados a continuación:

\section{(a) Categorización: deontología médica y confidencialidad}

Núcleo de sentido: relación médico-paciente, modelo humanista y biopsicosocial del curso de medicina de la ESCS, estimulador de la sensibilidad ética.

Del análisis de contenido de la entrevista, se concluyó que el $70 \%$ de los estudiantes demostraron comprender lo que sería necesario para tener actitudes éticas, presentando respuestas asertivas de acuerdo con el Código de Ética del Colegio Brasileño de Medicina. A partir de las observaciones de los ejemplos descritos a continuación y aportados en la categorización del problema 
(a), podremos interpretar la información recolectada: las percepciones, significados, explicaciones aportados por los propios protagonistas.

1(a) Ejemplos de respuestas asertivas de acuerdo con el Código de Ética del Colegio Brasileño de Medicina

( $1^{\mathrm{er}}$ año) “Elías tomó la actitud correcta, pues ayudó a preservar la salud de la esposa del paciente".

( $2^{\circ}$ año) “La actitud del médico se mostró sensata y adecuada a la situación, aunque haya visto que involucra riesgo de vida[...]"

( $3^{\text {er }}$ año " “[...]sería inhumano y una irresponsabilidad dejar a la esposa del portador de VIH expuesta al riesgo de contraer el virus".

( $4^{\circ}$ año) "Él actuó correctamente, ya que en este caso la omisión de los hechos coloca en riesgo la vida de otra persona".

( $4^{\circ}$ año) "El médico podría haber intentado convencer al paciente a informar él mismo a la esposa.

( $5^{\circ}$ año) "Yo intentaría convencer al paciente a revelar su enfermedad a la esposa y me colocaría a disposición para auxiliarlo[...]"

(Egresado de la $1^{\mathrm{a}}$ promoción del curso de medicina de ESCS) “[...]Elías reaccionó correctamente, pero debía de haberle contado a la esposa junto con el paciente[...]"

2(a) Ejemplos de respuestas que requieren una reflexión de acuerdo con el Código de Ética del Colegio Brasileño de Medicina

( $1^{\mathrm{er}}$ año) "La actitud del médico fue antiética, siendo que lo correcto sería convencer al paciente de lo importante que era que la esposa sepa de su enfermedad".

( $3^{\mathrm{er}}$ año) "Actitud equivocada, pues no guardó el principio de sigilo médico".

( $4^{\circ}$ año) "El médico está equivocado, pues es un secreto (sigilo) médico que fue pedido por el esposo para que él no lo revelase".

\section{(b) Categorización: ética médica y relación médico-paciente}

Núcleo de sentido: modelo humanista y biopsicosocial, estimulador de la sensibilidad ética. Construcción del vínculo, emoción y afectividad en la relación, con la vinculación del estudiante y el médico con el paciente, desarrollando la competencia técnica y la humanización en la relación médico-paciente, de acuerdo con la cultura latinoamericana. En las respuestas del $72 \%$ de los estudiantes de distintos años, se ha evidenciado poco miedo a la construcción de un vínculo emocional con el paciente y de la reflexión superficial de la ética.
1 (b)

Ejemplos de respuestas asertivas de los estudiantes de acuerdo con las directrices del Colegio Brasileño de Medicina y Ministerio de Educación

( $\left.1^{\mathrm{er}} \mathrm{año}\right)$ "El hombro amigo, desde que no sea exagerado, puede ayudar en la tentativa de amenizar el dolor de la madre".

( $\left.1^{\mathrm{er}} \mathrm{año}\right)$ "El estudiante quiso mostrarse solidario al sufrimiento de la madre cuando tomó una actitud humana".

( $3^{\mathrm{er}}$ año) "[...] la actitud del estudiante fue correcta, pues en las clases de comunicación en la ESCS, discutimos la importancia de la comunicación no verbal en la relación médico-paciente y la actitud del estudiante fue una tentativa de consuelo, que sería más difícil de ser expresada por el lenguaje verbal".

( $3^{\mathrm{er}}$ año) “[...]estamos delante de un conflicto entre el profesionalismo-racionalismo X humanismo, en que considero el humanismo, para tal situación, una forma adecuada para establecer una relación médico-paciente y que la actitud del médico en reprender al internado en público fue completamente inadecuada"

( $4^{\circ}$ año $)^{\text {“[ }[. . .] ~ e s e ~ c o n t a c t o ~ c o n ~ l a ~ m a d r e ~ e s ~ l a ~ f o r m a ~ d e ~ d a r ~ a p o-~}$ yo emocional a la misma"

(5 año) "El estudiante tuvo apenas una actitud de compasión... el profesor tiene una formación antigua, ultrapasada y rígida[... $]^{\prime \prime}$

( $5^{\circ}$ año) “[...] el paciente no necesita sólo medicamentos y sí afecto".

(Egresado de la $1^{\underline{a}}$ promoción del curso de medicina de la ESCS) “Cada persona tiene una forma e intensidad diferente de mostrar afecto y antipatía... soy como José en lo cotidiano, nunca perjudico la relación médico-paciente. Al contrario".

(Egresado de la $1^{\mathrm{a}}$ promoción del curso de medicina de la ESCS) “La postura del estudiante delante de la situación presentada fue de gran valor. Muchas veces el cariño vale más que cualquier receta médica".

(Egresado de la $2^{\underline{a}}$ promoción del curso de medicina de la ESCS) "La función del médico es cuidar de su paciente. A veces estos cuidados pueden darse a través de una palabra amable o de un gesto de afecto y cariño con su paciente, pues antes de ser pacientes o médicos, somos seres humanos"

\section{2 (b).Ejemplos de respuestas que requieren una reflexión de acuerdo con el Código de Ética Brasileño y las directrices curriculares nacionales para la carrera de medicina \\ ( $\left.1^{\mathrm{er}} \mathrm{año}\right)$. “El contacto físico con el paciente puede acercarnos.} El profesor tomó la actitud conforme le enseñaron y le orientaron durante su formación. Para él, debe existir distancia entre médico y paciente, lo que mantiene el respeto". 
(c) Categorización: procedimientos éticos en la enseñanza e investigación médica (con cadáver)

Núcleo de sentido: Ética del hospital-escuela. El paciente y el cadáver empleado en experimentos; estrés en la enseñanza-asistencia, tanatología, respeto al muerto y relación médico-enfermo. En cuanto a las actitudes éticas, el 57\% de las afirmaciones demuestra que los estudiantes del $1^{\text {er }}$ al $4^{\circ}$ año del curso, posiblemente no suscitarían actitudes éticas en sus acciones profesionales pues estarían prioritariamente preocupados con el tecnicismo y el aprendizaje práctico de la medicina.

1(c) Ejemplos de respuestas asertivas de los estudiantes de acuerdo con las directrices del Colegio Brasileño de Medicina y del Ministerio de Educación.

( $1^{\text {er }}$ año): “[...]realizar prácticas con un cadáver no es ético o responsable. La agonía de pensar en una situación en que un familiar le cuestione, como la propuesta, es la prueba de ello".

(3er año) "[...]reconocería mi error y pediría disculpas"

( $4^{\circ}$ año) "Explicar la situación (contando la verdad) y pedir autorización para realizar el procedimiento[...]"

(Egresado de la $1^{\text {a }}$ promoción del curso de medicina de ESCS). "[...]me coloco en el lugar de la hija y no me gustaría ver que el cadáver de mi padre sea utilizado como objeto[...]"

(Egresado de la 1aㅡ promoción del curso de medicina de ESCS) "Juan debía explicar lo que estaba haciendo, reconociendo su error, una vez que al utilizar el cadáver para tal objetivo sin el consentimiento de la familia es, aparte de antiético, ilegal"

(Egresado de la $2^{a}$ promoción del curso de medicina de ESCS) "Juan jamás podrá tomar tal actitud sin la autorización de la familia. Si él se siente inseguro ante tal situación, deberá acudir a un superior y pedir auxilio para desarrollar esas acciones."

\section{2 (c)Ejemplos de respuestas que requieren una reflexión de} acuerdo con el Código de Ética Brasileño y las directrices curriculares nacionales para la carrera de medicina.

( $1^{\text {er }}$ año): “A pesar de no ser malo, mentirle a la hija del paciente, era una forma de no causar más frustraciones en la persona que ya estaba abalada psicológicamente."

( $1^{\mathrm{er}}$ año): "Juan diría: estoy cuidando de su padre, por favor espere en la recepción. Después de este episodio, Juan podría salir del cuarto y dar la noticia a la persona que le aguarda."

( $1^{\text {er }}$ año): "Diría que estaba realizando procedimientos de costumbre para detectar la causa de la muerte."

( $1^{\text {er }}$ año): “En el lugar de Juan, utilizaría el cadáver para realizar procedimientos solamente con la autorización de un superior"
( $1^{\mathrm{er}}$ año): “Diría que estaba realizando procedimientos de rutina."

( $1^{\mathrm{er}}$ año): "No sé. Pensaría en mentir, pero creo que no sea lo correcto."

( $2^{\circ}$ año $)$ “[...] hablaría que [...] tales procedimientos eran obligatorios antes de la liberación del cuerpo[...]"

( $3^{\text {er }}$ año) “Esta actitud probablemente no sea políticamente correcta, pero no veo problemas ya que cualquier clase de anatomía se realiza en centros de necropsias con cadáveres. [...] Hablaría con la mayor naturalidad lo que estaba haciendo, a fin de que el familiar considere la actitud natural tratándose de un hospital escuela."

( $3^{\text {er }}$ año) “[...] Juan debía [...] disfrazar la situación creada y no relatar la verdad de su actitud lamentable."

( $4^{\circ}$ año " “...] haría de cuenta que los procedimientos son de rutina...si hablase la verdad, la hija se quedaría furiosa [...] desde que no haya falta de respeto al cadáver, no habría problemas en tomar ese tipo de conducta."

(d) Categorización: ética y respeto en la relación multidisciplinaria.

Núcleo de sentido: Impregnación de la práctica médica viciada, cooperativismo, dificultad en trabajar con la interdisciplinaridad, displicencia en cuanto a la postura ética. Se ha considerado en las respuestas la reflexión bajo la perspectiva de algunos valores como: información, secreto, respeto, confianza, justicia, autonomía, dignidad, veracidad, consentimiento, compromiso y los principios básicos de la bioética: autonomía, justicia, beneficencia, no maleficencia, sin jerarquización prioritaria de valores. Cerca del $90 \%$ de los estudiantes demostró actitudes asertivas cuanto a la relación ética y respetuosa con los demás profesionales de salud.

\section{1(d)Ejemplos de respuestas asertivas de los estudiantes de acuerdo con las directrices del Colegio Brasileño de Me- dicina}

( $1^{\mathrm{er}}$ año) “Jorge reaccionó como un ignorante, sin educación y sin ética. La falta de médico para tales procedimientos no es culpa de la enfermera, que apenas trataba de proveer las necesidades de la población. Esta situación debe ser tratada partiendo de principios de educación y respeto."

( $2^{\circ}$ año) “[...] Jorge debía de haber conversado con la enfermera particularmente y tratado directamente con ella para saber si era apta para continuar ejerciendo esas tareas que no le competían".

( $3^{\text {er }}$ año) "Si él hubiese reaccionado de una manera más educada, quizás la enfermera lo habría atendido." 
( $3^{\mathrm{er}}$ año) "Acusarla de incompetente no fue una buena alternativa. Se crea un clima pesado y desagradable en el equipo, que es interdisciplinario, perjudicando el ambiente de trabajo."

( $4^{\mathrm{o}}$ año) "Jorge está actuando corporativamente, defendiendo los intereses de su profesión, pero al mismo tiempo expresa prejuicio contra profesiones no medicas [...] debía haberse informado y tratado mejor a la enfermera."

( $5^{\circ}$ año) "Jorge se equivocó al no saber tratar de forma correcta a un colega de trabajo."

(Egresado de la 1ํㅡㄹ promoción del curso de medicina de la ESCS). "Jorge debe hacer eso (explicar que los procedimientos son exclusivos del médico) en un diálogo particular con la enfermera, y debe mostrarle respeto, a ella y a su trabajo".

(Egresado de la $2^{\underline{a}}$ promoción del curso de medicina de la ESCS). "Jorge tuvo una conducta antiética, pues humilló a una empleada y colega de trabajo en público, aparte de ofenderla cuando ella lo llamó de incompetente". de los recursos también clasifican la integración de la ética como muy integrada.

Se observó en el core curriculum una mayor inserción de la ética en el $1^{\text {er }}$ y $2^{\circ}$ año, cuando comparado a la inserción en el $3^{\text {er }}$ y $4^{\circ}$ año, y el internado, (IC95\%, á=0,034, pvalue=0,007), (Gráficos 1 a 3).

Asimismo, todos los años y las actividades educacionales como los módulos temáticos, habilidades y actitudes e interacción de la enseñanza con la comunidad tenían grados variables de inserción de la ética integrada en el curriculum.

Con relación a esta temática, la unidad de habilidades y actitudes fue la unidad educacional que presentó mayor recurrencia de los términos en el curriculum de los años $1^{\mathrm{er}}$ a $4^{\circ}$, destacándose el $2^{\circ}$ año seguido por el $4^{\circ}$ año. Los que presentaron mayor inserción fueron el $2^{\circ}$ y el $4^{\circ}$ año (Gráfico 1$)$.

Gráfico 1.

Evaluación de la inserción de los descriptores sobre Ética en el curriculum del $1^{\mathrm{er}}$ al $4^{\circ}$ año del Curso de Medicina de

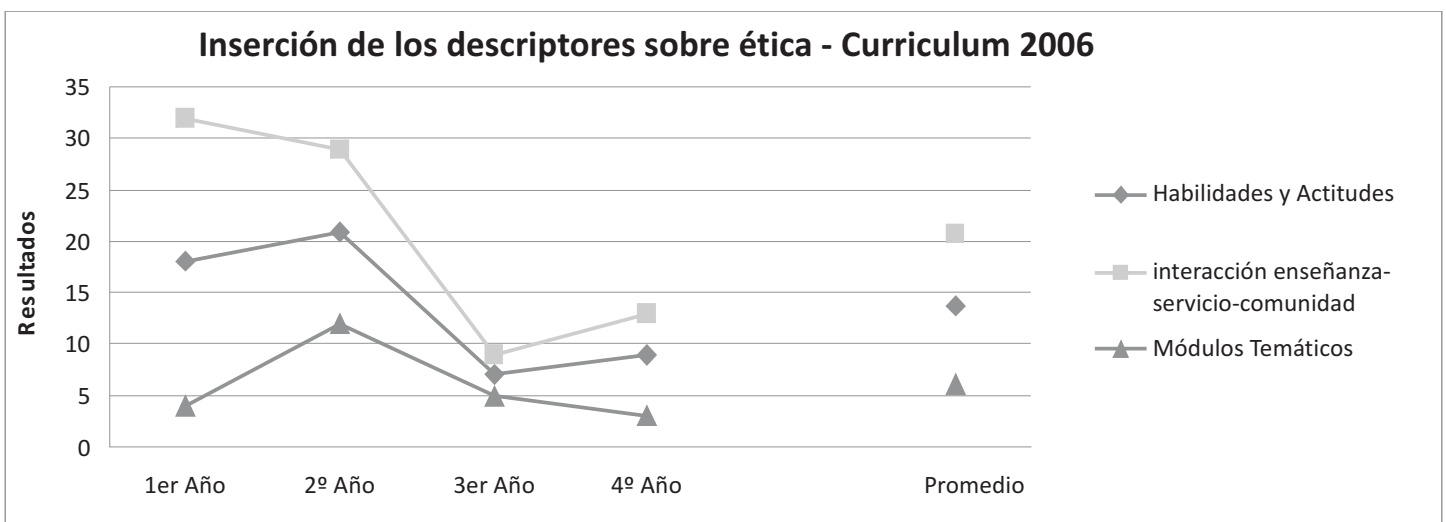

2 (d)Ejemplos de respuestas que requieren una reflexión de acuerdo con el Código de Ética Brasileño

( $1^{\mathrm{er}}$ año) “La enfermera está ejerciendo la medicina sin capacitación técnica y sin derecho legal, de esta forma ella debía ser denunciada"

( $1^{\mathrm{er}}$ año) "Jorge debe enterarse de las obligaciones y funciones de cada profesional de salud del PSF, para evitar tales inconvenientes"

( $2^{\circ}$ año) “Jorge no está equivocado cuanto a la competencia de la profesional, pero su abordaje debe ser menos áspero (ríspido...)"

En la segunda fase de la investigación se observó que el tema de la ética esta inserto en todas las actividades de los seis años. Otros parámetros del criterio SPICES, como la reducción de la fragmentación, objetivos de aprendizaje más altos de la taxonómica de Bloom, integración entre los docentes y racionalización
La unidad educacional interacción enseñanza-servicio-comunidad (IESC) del $3^{\text {er }}$ y $4^{\circ}$ año presentaron un pequeño abordaje de la ética en el curriculum del año de 2006, comparado al $1^{\mathrm{er}} \mathrm{y}$ $2^{\circ}$ año, que presentaron recurrencias más expresivas (Gráfico 1 ).

La unidad de habilidades y actitudes profesionales fue la unidad que presentó mayor recurrencia de la temática humanización, ética y bioética en el currículo del $1^{\mathrm{er}}$ al $4^{\circ}$ año (IC95\%-á=0,026, pvalue=0,013).

Cuando se comparan los datos obtenidos en el currículo, aplicados en el año 2001, a los resultados de la investigación desarrollada en el currículo aplicado en 2006, utilizando los mismos descriptores sobre ética y bioética, se constató que el currículo aplicado en 2001 presentó una menor recurrencia respecto a dos indicadores, al momento de comparar el desarrollo en el año de 2006 (Gráficos 2 y 3). 
Gráfico 2.

Evaluación de la inserción de los descriptores sobre Ética en el currículo aplicado del $1^{\text {er }}$ al $4^{\circ}$ año en 2001, en el

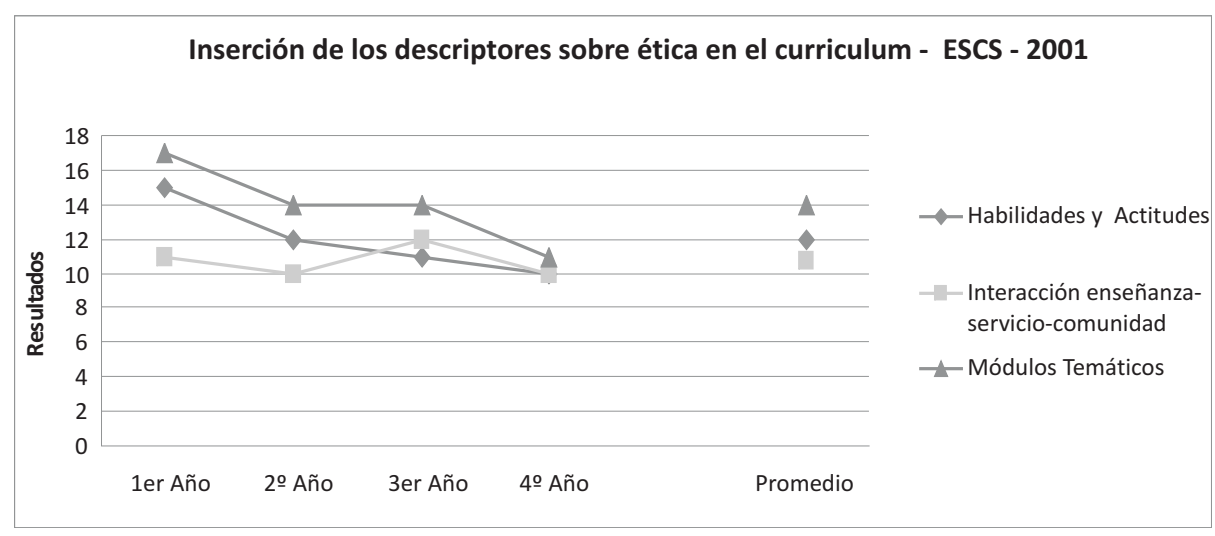

Gráfico 3.

Evaluación de la inserción de los temas de Ética en el Currículo de internado del Curso de Medicina de ESCS en

Inserción sobre ética en el curriculos del internado en los años de 2001 y 2006

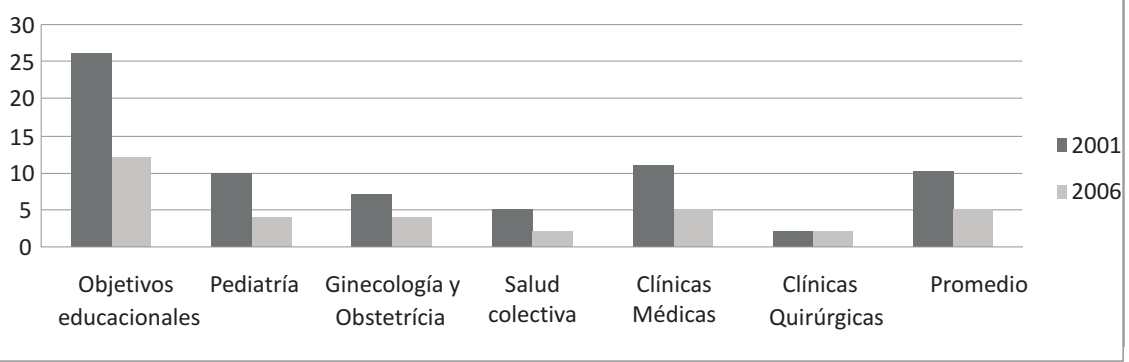

El internado médico es la última fase del pregrado en Medicina de la ESCS y corresponde al $5^{\circ}$ y $6^{\circ}$ año de curso, en que el estudiante participa de pasantías supervisadas por profesores y médicos especialistas en distintas áreas profesionales como Pediatría, Ginecología, Obstetricia, Clínicas Médicas y Clínicas Quirúrgicas, con 24 meses de duración, a partir del $5^{\circ}$ año. Los mismos descriptores usados anteriormente se analizaron en el programa de internado, concerniente a 2006.

Se observó la inserción de las temáticas de Ética y Bioética en el plan curricular y en las directrices generales del programa de internado, con excepción del programa de cirugía general, probablemente porque se da mayor énfasis a las habilidades específicas del área de actuación.

Este resultado ha sido mayor que lo planeado en el Plan Pedagógico del año de 2001. Cuando comparada la inserción entre el internado y las cuatro primeras series, se observa que en estas, la inserción de la ética y bioética es significativamente superior (IC95\%-á=0,042, pvalue=0,029).

\section{DISCUSIÓN}

La introducción de las temáticas de ética en la educación médica contribuyen a formar médicos capaces de acoger al paciente en su dimensión biopsicosocial y no solamente a buscar la cura de las enfermedades.

No existe un consenso relativo a cuál metodología de enseñanza sería la mejor para aprender Ética. Algunos autores afirman que la instrucción debe ocurrir utilizando métodos de aprendizaje activos, con discusión de dilemas bioéticos contextualizados en los diferentes campos de la ética social, deontológico, de la salud, y que contemple los avances científicos que repercuten en la sociedad ${ }^{4-6}$.

Algunos autores defienden que esta discusión debería ocurrir durante el progreso del curso, iniciándose en el primer año con la discusión de cuestiones de conflictos éticos, morales y de actitudes del estudiante delante del paciente, familiares y otros profesionales de la salud ${ }^{4-6,34-36}$. 
Asimismo, las actividades de contenido ético deberían estar integradas en los ciclos básicos y profesionales, cuando el proyecto pedagógico posibilite la integración curricular. Otros autores defienden que el contenido necesita estar relacionado a la práctica, que debe administrarse, preponderantemente, durante el ciclo de profesionalización en los currículos tradicionales ${ }^{4-6,34-36}$

En 2001, las 103 escuelas de Medicina brasileñas presentaban la Ética inserta en sus currículos. Los métodos de enseñanza incluían clases expositivas, discusión de casos, presentación de seminarios, entre otros ${ }^{41}$.

Comparando con los datos de 1992, es posible afirmar que hubo de hecho una expansión de la enseñanza de la Ética y Bioética. Sin embargo, al analizarse la carga horaria en ambos períodos, se observa un aumento muy pequeño. Actualmente, existe una interesante tendencia de la valoración de los elementos de la Ética, cuyos resultados futuros serán, indudablemente, positivos ${ }^{41}$.

En las escuelas médicas, la construcción de la postura ética y del pensamiento crítico y reflexivo debería desarrollarse a partir de las actividades interdisciplinarias con la humanización. Con el desarrollo de los contenidos que estuviesen relacionados con la medicina, entre ellos los provenientes de las áreas de filosofía, ética, psicología, antropología, artes, sociología, historia, política. Estos vínculos podrían llegar a formar profesionales con una visión más humanizada ${ }^{1-3,11,12}$.

Es necesario cambiar actitudes y comportamientos e incidir en la relación profesional de la salud-paciente y en los modelos de asistencia en salud. Cambiar actitudes en los estudiantes de medicina o en los profesionales es bastante más complejo ${ }^{20}$. Medir la actitud no es un proceso sencillo sino, que demanda la construcción de un instrumento especialmente diseñado para este fin. Un instrumento de medición adecuado es aquel que registra datos observables que representa verdaderamente los conceptos o variables que el investigador considera medir ${ }^{21}$.

Las clases magistrales, que emplean los métodos de enseñanza tradicional, pueden ayudar a transmitir contenidos de forma pasiva al estudiante, pero cuando se trata de cambiar actitudes y transmitir valores resultan bastante menos eficaces que el trabajo en grupos pequeños interactuando con el profesor. Para el cambio de actitudes, el estudiante debe estar en contacto permanentemente con acciones educativas:

1. Aprendizaje ético-clínico, prácticas o caso que le haya sucedido y tutorías en pequeños grupos, fundamentando el aprendizaje en casos que descubran no sólo métodos de resolución y decisión, sino actitudes en los pacientes y en los equipos de salud, estimulando el trabajo interdisciplinario.
2. Usar el método del aprendizaje basado en problemas para cambio de actitudes, este método es usado en el Curso de Medicina de la ESCS. El autor sugiere aún unir el aprendizaje clínico con el aprendizaje ético en tutorías de grupos pequeños, en las cuales se complementa la resolución clínica del caso con la resolución de los problemas ético-clínicos que van apareciendo.

3. Otra sugerencia presentada es utilizar métodos audiovisuales diversos que acerquen a los alumnos a los problemas reales de la sociedad. Es un instrumento para la enseñanza que pretende acercarse a las interrogantes y a la vida de los estudiantes $^{42}$

4. Usar también comentarios de noticias o informes de prensa, casos reales que hayan sido analizados por los comités de bioética, con la debida confidencialidad, material didáctico específico y claro, entre otros recursos ${ }^{43}$.

En la etapa de internado o de residencia es especialmente importante que los estudiantes puedan dialogar con los tutores sobre los aspectos éticos de la clínica. Los estudiantes intentar cambiar sus actitudes si ven también cambios en sus docentes, mediante la observación de la coherencia entre lo que se habla y lo que se practica en la relación profesor-alumno ${ }^{43}$.

Además, un bagaje humanístico permitirá a los médicos una mejor comprensión del pasado, un mejor análisis del presente y una mejor visión del futuro y así lograr una perspectiva independiente de la tecnología, sin restarle a ésta el indudable valor que tiene, pero en la medida correspondiente. Nos permitirá seguir asociando el juicio humano a nuestras decisiones técnicas, en una armonía absolutamente deseable para este mundo postmoderno ${ }^{50}$.

En este trabajo se ha evidenciado frecuencias diferentes de los temas relacionados con la ética en el currículo de dos cursos de medicina brasileños, así como una distribución de estos temas en diferentes períodos del curso. ¿Será que la mayor carga horaria y discusión de una variedad de temas sobre bioética resultan en médicos más éticos? No se puede tener certeza sobre la formación de estos médicos, las escuelas deben incluir reflexiones que posibiliten la formación ética y humanista de los egresados y contribuir a una mejor asistencia médica a la sociedad brasileña.

\section{CONCLUSIÓN}

La universidad actual es la encargada de formar profesionales capaces de asimilar los cambios tecnológicos e introducirlos en la práctica social de manera ética, creativa e innovadora; esto exige transformaciones en el proceso de formación del profesional que responda a la dinámica de cambio cultural del mundo para este siglo. 
En la Educación Superior el Diseño Curricular ha pasado por diferentes etapas y en estos momentos es objeto de reevaluación. Las directrices curriculares nacionales e internacionales para el pregrado en medicina, legitimaron las bases para la construcción de los currículos objetivando el aprendizaje de conocimientos, la formación de habilidades y percepción de valores éticos, insertos en el contexto de humanidades y profesionalismo.

El core curriculum desarrollado en 2006 en la carrera de medicina en la Escuela Superior en Ciencias de la Salud - ESCS, una escuela brasileña que utiliza en su plan curricular metodologías activas de enseñanza como Aprendizaje Basado en Problemas, problematización y la aplicación del Arco de Maguerez en la resolución de los problemas de salud, contempló la temática de la ética integrada en todas los años de la carrera e internado.

Los egresados de la carrera de la ESCS demostraron manejar bien los conflictos éticos y respeto a los pacientes. Sin embargo, el análisis de la sensibilidad ética denunció fragilidad en las percepciones y actitudes inapropiadas de los estudiantes de la carrera de medicina, identificada especialmente en los años iniciales.

Los autores de esta investigación recomiendan el desarrollo de más discusiones sistematizadas del tema en los cursos incluyéndose los recursos didácticos de las metodologías activas de enseñanza, mediante la discusión de películas actuales, informes de revistas científicas y telenoticias, relacionando materias y documentos actuales a los temas y conceptos de bioética en general, además de la ética profesional discutida en las actividades prácticas del plan curricular de la carrera de medicina, para que haya un crecimiento y fortalecimiento de la reflexión ética de los estudiantes.

Una mirada ética y humanística permitirá a los médicos una mejor comprensión del pasado, un mejor análisis del presente y una mejor visión del futuro, y así lograr una perspectiva independiente de la tecnología, asociando el juicio humano a las decisiones técnicas.

\section{REFERENCIAS}

1. Tonelli E. Escolas Médicas. Jornal da Associação Médica de Minas Gerais. Dez 2004 - jan. 2005:p.7.

2. Povoa L, Andrade MV. Distribuição geográfica dos médicos no Brasil; uma análise a partir de um modelo de escolha locacional. Cad Saúde Pública. 2006;8:1555-64.

3. Brasil. Ministério da Educação. Conselho Nacional de Educação. Câmara de Educação Superior. Resolução CNE/CES n.ำ 4, de 7 de novembro de 2001. Institui diretrizes curriculares nacionais do curso de graduação em medicina. Diário Oficial da União. Brasília, 9 nov. 2001; Seção 1, p.38.
4. Feuerwerker LCM. Mudanças na educação médica e residência médica no Brasil. São Paulo: Hucitec; Rede Unida; 1998.

5. Goldie J. The impact of three years' ethics teaching, in an integrated medical curriculum, on students' proposed behaviour on meeting ethical dilemmas. Med Educ. 2002;36:489-97.

6. Tyler R. Basics principles of curriculum and instruction. Chicago: University of Chicago Press; 1950.

7. Flexner A. Medical education in the United States and Canada. A report to the Carnegie Foundation for the Advancement of Teaching. Boston, Massachusetts: Updyke; 1910.

8. Taba H. Elaboración del currículo: teoría y práctica. Buenos Aires, Argentina: Editorial Troquel; 1983.

9. Muñoz D, Muñoz DR. OEnsino da Ética nas Faculdades de Medicina do Brasil. Rev Bras Educ Med. 2003;27(2):114-24.

10. Schanaider A. Integração das ciências básicas e áreas profissionais no ensino de graduação em medicina. Rev Bras Educ Med. 2002;26(1):67-70.

11. Hebert PC, Meslin EM, Dunn EV. Measuring the ethical sensitivity of medical students: a study at the University of Toronto. J Med Ethics 1992;18:142-147.

12. Association of American Medical College. Learning Objectives for Medical Student Education: Guidelines for Medical Schools [online]. 1998 [acess 2007 oct. 12]. Available: https://services.aamc.org/Publications/index.cfm?fuseaction=Product.displayForm\&prd_id=198\&prv_id=239.

13. General Medical Council. Tomorrow's doctors (2003). Regulating doctors ensuring good medical practice [online]. 2003 [acesso em: 2007 oct. 12]. Available: http://www.gmc-uk.org/static/documents/content/TomorrowsDoctors_2003.pdf

14. Association for Medical Education in Europe [homepage]. Scotland, UK: AMEE; c2008- [acess 2008 sep. 10]. Available: http:/ /www.amee.org.

15. BRASIL. Ministério da Saúde. Ministério da Educação. Portaria Interministerial n.․ 610, de 26 de março de 2002. Institui o programa nacional de incentivo às mudanças curriculares para as escolas médicas, com o objetivo de incentivar a promoção de transformações dos processos de formação, geração de conhecimentos e prestação de serviços a comunidade, por meio de inovações curriculares, baseadas nas diretrizes curriculares para os cursos de medicina, aprovadas pelo Ministério da Educação [online]. [acesso em 10 set. 2008]. Disponível em: http: / / www.promed.unifesp.br/pdf/projeto_2002.pdf 
16. Miles SH, Lane LW, Bickel J, Walker RM, Cassel CK. Medical ethics education: coming of age. Acad Med. 1989;64:705-9.

17. Self DJ, Wolinsky FD, Baldwin DC. The effect of teaching medical ethics on medical students' moral reasoning. Acad Med. 1989;64:755-9.

18. López Guzmán J. Ética en la industria farmacéutica: entre la economía y la salud. Pamplona: Universidad de Navarra; 2005.

19. Jaime OMR. Exploración y aplicación de conocimientos bioéticos en el personal de enfermería. Rev Cubana Enfermer. 2003;19(3).

20. Cantillo J, Domínguez A, Encinas S. Dilemas Morales. Un aprendizaje de valores mediante el diálogo. Valencia: Nau Llibres;2005.

21. Outomuro D. Fundamentación de la enseñanza de la bioética en medicina. Acta Bioeth. 2008; 14(1):19-20.

22. Rego S. A formação ética dos médicos: saindo da adolescência com a vida (dos outros) nas mãos. Rio de janeiro: Ed. Fiocruz; 2003.

23. Correa FJL. Enseñar bioética: cómo trasmitir conocimientos, actitudes y valores. Acta Bioeth. 2008;14(1):11-18.

24. Rodriguez Yunta E. Ética e innovación tecnológica. Santiago de Chile: CIEB Universidad de Chile; 2006.

25. Kottow M, Alvarez C, Santander P, Urrutia P, Cumsille F, Roa A. Cambios de actitudes éticas a lo largo de los estudios de medicina. Rev Med Chile. 1993;121(4):379-84.

26. Buendía A, Alvarez de la Cadena C. Nivel de conocimiento de la bioética en carreras de odontología de dos Universidades de América Latina. Acta Bioeth. 2006;12(1):41-6.

27. Rennie S, Rudland J. Differences in medical students' attitudes to academic misconduct and reported behaviour across the years: a questionnaire study. J Med Ethics. 2003;29(2):97-102.

28. Epstein RJ. Learning from the problems of problem-based learning. BMC Med Educ [periódico na Internet]. 2004 [acess . 2008 jan. 5];4(1): [aproximadamente 9p.]. available http: / / www.biomedcentral.com/content/pdf/1472-6920-4-1.pdf

29. Batista SHS. A interdisciplinaridade no ensino médico. Rev Bras Educ Med. 2006;30(1):39-46.

30. Mofidi M, Strauss R, Pitner LL, Sandler ES. Dental Students' Reflections on Their Community-Based Experiences: The Use of Critical Incidents. J Dent Educ. 2003;67(5):515-23.
31. Rennie S, Crosby J. Are "tomorrow's doctors" honest? Questionnaire study exploring medical students' attitudes and reported behaviour on academic misconduct. BMJ. 2001;322:274-5.

32. División de Salud y Desarrollo Humano. Coordinación de Investigaciones. Programa de Subvenciones para la Investigación. Normas Éticas del PSI para investigaciones con Sujetos Humanos. Washington: HDP/HDR/PSI; OPS/OMS; 1996.

33. Borrell-Carrió F, Suchman AL, Epstein RM. The biopsychosocial model 25 years later: principles, practice, and scientific inquiry. Ann Fam Med. 2004;2(6):576-82.

34. Trindade E. Resgatando a dimensão subjetiva e biopsicossocial da prática médica com estudantes de medicina: Relato de caso. Rev Bras Educ Med. 2006;29(1):48-50.

35. Silverthorn DU. Teaching and learning in the interactive classroom. Adv Physiol Educ [periódico na Internet]. 2006 [acess 2007 dec. 18];30: [aproximadamente 5p.]. available: http:/ /advan.physiology.org/cgi/content/full/30/4/135

36. Penaforte JC. John Dewey e as raízes filosóficas da aprendizagem baseada em problemas. In: Mamede S, Penaforte J. orgs. Aprendizagem baseada em problemas: anatomia de uma nova abordagem educacional. Fortaleza: Hucitec; 2001.

37. Mclean M, Van Wyk JM, Peters-Futre EM, Higgins-Opitz $\mathrm{SB}$. The small group in problem-based learning: more than a cognitive 'learning' experience for first-year medical students in a diverse population. Med Teach [periódico na Internet]. 2006 [acess 2007 dec. 20];28(4): [aproximadamente 10p.]. available: http://taylorandfrancis.metapress.com/media/c28459nnth2qxka1tdtx/contributions/r/6/6/1/r6610v7p428x47rw.pdf>

38. Fundação de Ensino e Pesquisa em Ciências da Saúde. Secretaria de Estado de Saúde. Projeto Pedagógico do Curso de Medicina da Escola Superior em Ciências da Saúde. Brasília: FEPECS/SES; 2001.

39. Bussad WO, Morettin PA. Estatística Básica. 5ae ed. São Paulo: Saraiva; 2003.

40. Blaikie NWH. A critique of the use of triangulation in social research. Quality and Quantity 1991; 25:115-36.

41. Paul J. Between Method Triangulation. The International Journal of Organizational Analysis. 1996; 4(2):135-53.

42. Institute for International Medical Education. [homepage]. New York: IIME; c1999-2006 [Revised 2002; acess 2008 
oct. 02]. Glossary of Medical Education Terms; [1 tela]. available: http://www.iime.org/glossary.htm.

43. Harden, RM. Educational strategies in curriculum development: the SPICES model. Med Educ. 1984;18(4):284-297.

44. Van den Berg H. Rating of SPICES criteria to evaluate and compare curricula. Med Teach. 2004;26(4):381-3.

45. Bardín L. Analisis de Contenido. Madri: Akal; 1986.

46. Pontes AL, Rego S, Silva Junior AG. Saber e prática docente na transformação do ensino médico. Rev Bras Educ Med. 2006;30(2):66-7.

47. Tomas MC, Tomás GM. La vida humana a través del cine. Cuestiones de antropología y bioética. Madrid: Eiunsa; 2004.

48. Lolas F. Bioética. Santiago de Chile: Universitaria; 1998

49. Gracia D. La deliberación moral, el método de la ética clínica. Med Clín (Barc.) 2001;117(1):18-23.

50. Norero VC. Humanismo y Medicina. La proyección humanista en la enseñanza de la Medicina chilena del siglo XXI. Rev Chil Pediatr. 2003;74(3):253-5.

\section{CONTRIBUCION DE LOS AUTORES}

Maria Rita C. G. Novaes participó en la elaboración del proyecto, recopilación de datos, análisis e interpretación de los resultados, redacción y revisión final del texto. Luiz Carlos G. Novaes participó en el análisis e interpretación de los resultados, redacción y revisión final del texto. Dirce Guilhem participó en el análisis e interpretación de datos, redacción y revisión final del texto. Carla Cristina C. Silveira y Murilo G. Guiotti participaron en la recopilación de datos y tabulación de los mismos para el análisis estadístico y de contenidos. Eliane M. V. Trindade participó en la elaboración del proyecto, análisis e interpretación de los resultados cualitativos, redacción y revisión final del texto.
Ricardo Komatsu y Fernando Lolas participaron la elaboración del proyecto, redacción y revisión final del texto.

\section{CONFLICTO DE INTERESES}

Declaró que no hubo

\section{CORRESPONDENCIA}

Maria Rita Carvalho Garbi Novaes

SHIS-QI-09- conj. 06- cs14

Setor de Habitações - Lago Sul

CEP. 71625-060 DF

E-mail: ritanovaes@ig.com.br

\section{RESUMO}

Atitudes Éticas dos estudantes e egressos de medicina com metodologias ativas

O objetivo deste estudo foi desenvolver um diagnóstico da inserção integrada da ética na carreira de medicina brasileira com metodologia de aprendizagem baseado em problemas e descrever as percepções de atitudes éticas que tem os estudantes e egressos. O desenho metodológico é um estudo de caso, descritivo e documental, com abordagem qualitativa e quantitativa. A amostra desta investigação constituiu-se por 20 (vinte) estudantes / ano, totalizando de 120 estudantes e 40 egressos de duas turmas do Curso de Medicina da ESCS. Este projeto foi aprovado pelo Comitê de Ética em Pesquisa - SES/DF.

Os estudantes e egressos da ESCS demonstraram abordar os conflitos éticos e o respeito aos pacientes. Contudo, a análise da sensibilidade ética denunciou fragilidade quanto às percepções e atitudes inapropriadas dos estudantes da carreira de medicina, identificada especialmente nos anos iniciais, necessitando de mais discussões sistematizadas sobre os aspectos éticos e bioéticos integrados às atividades práticas, para que haja crescimento e fortalecimento da reflexão ética dos estudantes. 